\title{
MOND rotation curves for spiral galaxies with Cepheid-based distances
}

\author{
R. Bottema ${ }^{1}$, J. L. G. Pestaña ${ }^{2}$, B. Rothberg ${ }^{3}$, and R. H. Sanders ${ }^{1}$ \\ 1 Kapteyn Astronomical Institute, PO Box 800, NL-9700 AV Groningen, The Netherlands \\ 2 Dpto. de Física, Univ. de Jaén, Virgen de la Cabeza 2, 23071-Jaén, Spain \\ 3 Institute for Astronomy, 2680 Woodlawn Dr., Honolulu, HI 96822, USA
}

Received 18 February 2002 / Accepted 9 July 2002

\begin{abstract}
Rotation curves for four spiral galaxies with recently determined Cepheid-based distances are reconsidered in terms of modified Newtonian dynamics (MOND). For two of the objects, NGC 2403 and NGC 7331, the rotation curves predicted by MOND are compatible with the observed curves when these galaxies are taken to be at the Cepheid distance. For NGC 3198, the largest distance for which reasonable agreement is obtained is $10 \%$ smaller than the Cepheid-based distance; i.e., MOND clearly prefers a smaller distance. This conclusion is unaltered when new near-infrared photometry of NGC 3198 is taken as the tracer of the stellar mass distribution. For the large Sb spiral, NGC 2841, MOND requires a distance which is at least 20\% larger than the Cepheid-based distance. However, the discrepancy of the Tully-Fisher and SNIa distances with the Cepheid determination casts some doubt upon the Cepheid method in this case.
\end{abstract}

Key words. galaxies: individual: NGC 3198, NGC 2841 - galaxies: kinematics and dynamics - galaxies: spiral

\section{Introduction}

It is well established that, in the context of Newtonian dynamics, the observable mass in spiral galaxies cannot account for the observed flat rotation curves in the outer regions of galaxies (Bosma 1978; Begeman 1987; van Albada et al. 1985). The standard explanation for this discrepancy is the proposal that galaxies are embedded in an extended dark halo which dominates the gravitational field in the outer regions (Trimble 1987).

An alternative explanation for the discrepancy is the possibility that dynamics becomes non-Newtonian in the limit of low accelerations. The most successful such proposal is Milgrom's (1983) modified Newtonian dynamics or MOND. Here the idea is that below a certain acceleration threshold $\left(a_{0}\right)$ the effective gravitational acceleration approaches $\sqrt{a_{\mathrm{o}} g_{\mathrm{n}}}$ where $g_{\mathrm{n}}$ is the usual Newtonian acceleration. This modification yields asymptotically flat rotation curves of spiral galaxies and a luminosity - rotation velocity relationship of the observed form, $L \propto v^{4}$, the Tully-Fisher relation (Tully \& Fisher 1977). But apart from these general aspects the prescription also successfully predicts the observed form of galaxy rotation curves from the observed distribution of stars and gas with reasonable values for the mass-to-light ratio of the stellar component (Begeman et al. 1991; Sanders 1996; Sanders \& Verheijen 1998; McGaugh \& de Blok 1998). A crucial element of a very

Send offprint requests to: $\mathrm{R}$. H. Sanders, e-mail: sanders@astro.rug.nl specific prescription like MOND is that the precise form of the rotation curve is predicted by the observed mass distribution given the value of a single universal parameter; in this case, the critical acceleration $a_{0}$. Consequently MOND can in principle be falsified as soon as there is one galaxy for which the predicted rotation curve disagrees significantly with the observed curve; although, in practice, the usual uncertainties inherent in astronomical data render a definitive falsification problematic in any individual case.

In Begeman et al. (1991, hereafter BBS) MOND is applied to a sample of galaxies for which high quality $\mathrm{H}_{\text {I }}$ rotation curves are available. For a value of $a_{0}$ equal to $1.21 \times$ $10^{-8} \mathrm{~cm} \mathrm{~s}^{-2}$ the rotation curves of the sample could be reasonably reproduced, the free parameter in each case being the mass-to-light ratio of the visible disc. Because MOND is an acceleration dependent modification, this derived value of $a_{\mathrm{o}}$ depends upon assumed distance scale $\left(H_{\mathrm{o}}=75 \mathrm{~km} \mathrm{~s}^{-1} \mathrm{Mpc}^{-1}\right.$ in this case). Moreover, the quality of an individual fit depends upon the adopted distance to the galaxy, and, since the relative distances to these nearby galaxies have not been known to within an accuracy, typically, of $25 \%$, this has provided some freedom to adjust the distance in order to improve the MOND fit; i.e., distance, within certain limits, can be considered as an additional second parameter in the fitting procedure. For most of the galaxies in the sample of BBS, the distance did not have to be adjusted significantly $(<10 \%)$ to improve the MOND fits, and the improvement was not significant. However, one object, 
NGC 2841, required a large readjustment: the Hubble law distance to this galaxy is about $9 \mathrm{Mpc}$, but MOND clearly prefers a distance which is twice as large.

Using ground-based and Hubble Space Telescope observations Cepheid distances to 21 inclined galaxies have now been determined as part of the HST key program on the extragalactic distance scale (e.g. Sakai et al. 2000). Three of the galaxies in this Cepheid sample are also in the sample with high quality rotation curves considered by BBS. These are NGC 2403 (Freedman \& Madore 1988), NGC 3198 (Kelson et al. 1999) and NGC 7331 (Hughes et al. 1998). For these three galaxies the MOND prescription can now be considered in the context of the Cepheid distance that is generally considered to be the most precise indicator.

NGC 2841 has been discussed as a critical case for MOND by Sanders (1996). For this galaxy, there is also a large discrepancy between the Tully-Fisher distance and the Hubble law distance (for plausible values of the Hubble constant). Moreover, the galaxy was the site of a recent SNIa (1999by). For these reasons this galaxy has been included, subsequently, in the HST program (Macri et al. 2001).

Here we demonstrate that for two galaxies in the BBS sample the rotation curve predicted by MOND is consistent with the observed curve when the galaxies are placed at the Cepheid distance. However, for NGC 3198 at the Cepheid distance of $13.8 \pm 0.6 \mathrm{Mpc}$, the shape of the rotation curve predicted by MOND systematically deviates (by up to $10 \mathrm{~km} \mathrm{~s}^{-1}$ ) from the observed curve, both in the inner and outer regions. The largest distance which can be compatible with MOND is about $10 \%$ lower than the Cepheid-based distance. This is not particularly problematic because of likely uncertainties in the Cepheid method and in the determination of a rotation curve from the observed two-dimensional velocity field. NGC 2841, however, remains a difficult case for MOND. The minimum distance which is consistent with MOND is about $17 \mathrm{Mpc}$ whereas the Cepheid-based distance is $14.1 \pm 1.5 \mathrm{Mpc}$. We discuss the implications and seriousness of this discrepancy for MOND, or, alternatively, for the Cepheid method.

\section{Determination of the MOND rotation curve}

The procedure followed when determining a MOND rotation curve has been described previously (e.g., BBS). In the context of MOND, the true gravitational acceleration $g$, is related to the Newtonian acceleration $g_{\mathrm{n}}$ as

$\mu\left(g / a_{0}\right) g=g_{\mathrm{n}}$

where $a_{0}$ is the acceleration parameter and $\mu(x)$ is some function which is not specified but has the asymptotic behavior

$\mu(x)=1, \quad x>1$ and $\mu(x)=x, \quad x<1$

(Milgrom 1983); a convenient function with this asymptotic behavior is

$\mu(x)=x\left(1+x^{2}\right)^{-1 / 2}$.

The circular velocity is given as usual by

$v=\sqrt{r g}$.
From Eqs. (1), (2) and (4) it is evident that the rotation curve about a finite bounded mass $M$ in the low acceleration limit is asymptotically flat at a value given by

$v^{4}=G M a_{0}$,

which forms the basis of the observed Tully-Fisher (TF) relation. The Newtonian acceleration $g_{\mathrm{n}}$ is determined, as usual, by applying the Poisson equation to the mass distribution deduced from the distribution of the observable matter (disc, bulge, and gas). The surface density distribution of the stellar disc is assumed to be traced by the distribution of visible light (i.e., no variation of $M / L$ within a given component of a given galaxy), but then the question arises as to which photometric band is most appropriate. The near-infrared emission (e.g., $K^{\prime}$-band) is considered to be a better tracer of the old dominant stellar population, and less susceptible to positiondependent extinction, but this is not generally available. Below, we use the $r$-band as a tracer of the form of the mass distribution in the stellar disc, but, with respect to NGC 3198, we also consider more recent $K^{\prime}$-band photometry.

The stellar disc may be assumed to be asymptotically thin or have a finite thickness related to the radial scale length of the disc by an empirical rule (van der Kruit \& Searle 1981); this makes little difference in the final result. Applying Eqs. (1), (3), and (4), a least squares fit is then made to the observed rotation curve $v(r)$ where the single free parameter of the fit is the massto-light ratio of the disc; in cases where there is an indication of a bulge from the light distribution, $M / L$ of the bulge enters as a second parameter.

For the gaseous component a surface density distribution equal to that of the $\mathrm{H}_{\mathrm{I}}$ is taken, multiplied by a factor 1.3 to account for primordial helium. The gas layer is taken to be infinitesimally thin. The contribution of the gas to the total rotation is fixed, but does depend on the distance to the galaxy.

In principle, the parameter $a_{\mathrm{o}}$ should be universal and, having determined its magnitude, one is not allowed to adopt this as a free parameter. But as noted above, the derived value of $a_{\mathrm{o}}$ does depend upon the assumed distance scale. Sanders \& Verheijen (1998) give MOND fits to the rotation curves of 30 spiral galaxies in the UMa cluster which they assume to be at $15.5 \mathrm{Mpc}$. The preferred value of $a_{\mathrm{o}}$ with this adopted distance is equal to the BBS value of $1.2 \times 10^{-8} \mathrm{~cm} \mathrm{~s}^{-2}$. However, based upon the Cepheid-based re-calibrated TullyFisher relation (Sakai et al. 2000), Tully \& Pierce (2000) argue that the distance to UMa should be taken to be $18.6 \mathrm{Mpc}$. We have recalibrated the Tully-Fisher law using this same sample of galaxies but with the three test galaxies (NGC 2403, NGC 3198, NGC 7331) left out of the fitting. Within the errors, the slope and intercept of the Tully-Fisher relation are the same as that found by Sakai et al. (2000), and the distance to Ursa Major is only $1 \%$ smaller than that found Tully \& Pierce (2000). In that case the MOND fits to the UMa galaxies imply that the value of $a_{\mathrm{o}}$ should be adjusted to $0.9 \times 10^{-8} \mathrm{~cm} \mathrm{~s}^{-2}$. This is also the preferred value of $a_{\mathrm{o}}$ from MOND fits to rotation curves of a sample of nearby dwarf galaxies with distances taken primarily from group membership (Swaters \& Sanders 2002, in preparation). 

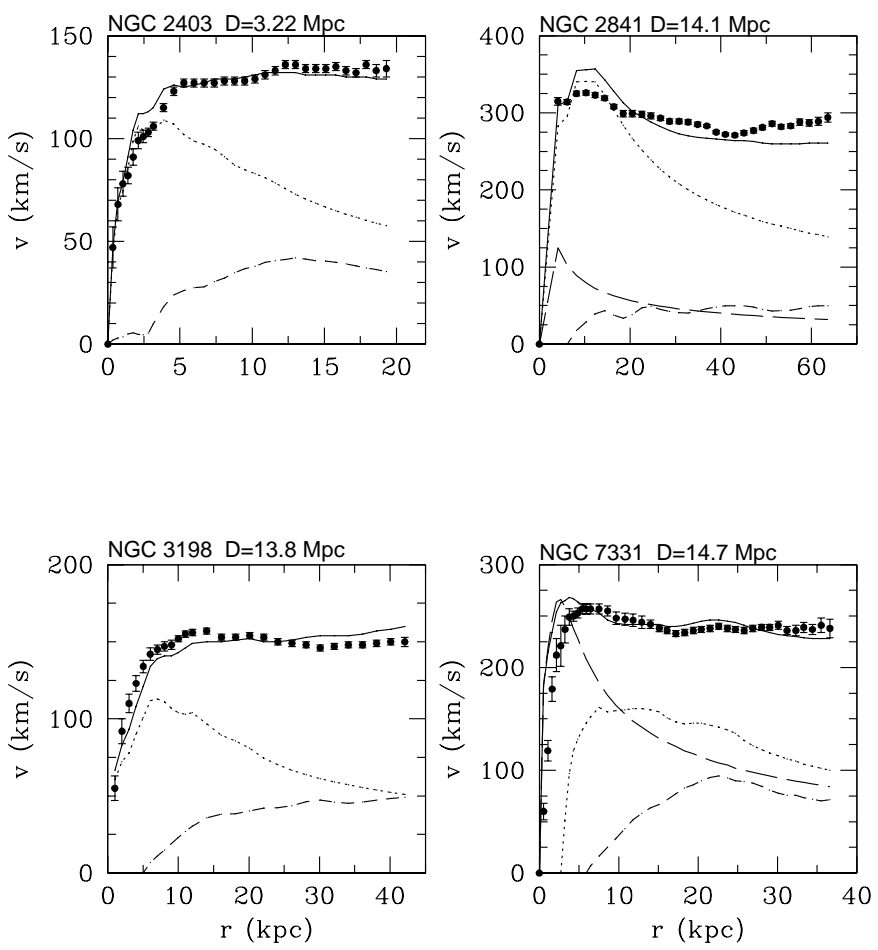

Fig. 1. MOND rotation curves compared to observed Hi rotation curves for the four galaxies from the sample of BBS with Cepheidbased distances. The dotted, long-dashed, and short-dashed lines are the Newtonian rotation curves of the stellar disc, bulge, and gaseous components respectively.

\section{Rotation curve fits at Cepheid-based distances}

In Fig. 1 we show the MOND rotation curve for all four galaxies from the BBS sample with Cepheid-based distance determinations. NGC 2841 and NGC 7331 both contain central bulges as evidenced in the light distribution, and the radial surface brightness profile has been appropriately decomposed. Here, $a_{\mathrm{o}}$ is fixed at the rescaled value of $0.9 \times 10^{-8} \mathrm{~cm} \mathrm{~s}^{-2}$, and the distance is fixed at the Cepheid-based values as updated and corrected by Freedman et al. (2001). The free parameters of the fit are the disc and, in two cases, bulge masses. The resulting values and the corresponding mass-to-light ratios are given in Table 1 for the four galaxies.

Here we see that for two of the galaxies, NGC 2403 and NGC 7331, the MOND rotation curves agree well with the observed curve. In both of these cases, the predicted rotation curve lies somewhat above the observed curve in the inner regions but this could be due to beam-smearing. The implied mass-to-light ratios generally fall within the range that would be considered reasonable for stellar populations (Table 1).

For the other two galaxies, there are clear systematic differences between the MOND rotation curve and the observed curves. Basically, the predicted curves have a different shape than the observed curves: for NGC 2841, the predicted curve is significantly higher than observed in the inner regions (by up to $30 \mathrm{~km} \mathrm{~s}^{-1}$ ) and comparably lower in the outer regions. For NGC 3198 the differences are in the opposite sense: about $10 \mathrm{~km} \mathrm{~s}^{-1}$ lower in the inner regions and $10 \mathrm{~km} \mathrm{~s}^{-1}$ higher in the outer regions. These differences diminish if NGC 2841 is moved further out and if NGC 3198 is moved closer in; i.e., MOND clearly prefers a larger distance to NGC 2841 (as discussed previously by BBS and by Sanders 1996) and a smaller distance to NGC 3198 . We now discuss these two cases with respect to the question of whether or not this mismatch can be interpreted as a falsification of MOND. Because the rotation curve of NGC 3198, when taken at the Hubble law distance of $10 \mathrm{Mpc}$, is very well predicted by MOND, and because the observed curve is thought to be well-determined, this, at first sight, appears to be the more problematic case, and we begin with this object.

\section{NGC 3198}

\section{1. $r$-band photometry}

This gas-rich spiral galaxy has a generally symmetric $\mathrm{H}$ I distribution, and there are no large scale significant warps or distortions of the velocity field. The rotation curve extends to roughly 10 radial scale lengths and is, to first order, flat and featureless (Begeman 1987). For these reasons it has become the classic case of a spiral galaxy evidencing a large mass discrepancy in its outer regions (van Albada et al. 1985). If any theory, such as MOND, fails to predict the rotation curve of this galaxy, then it would be problematic for that theory.

In Fig. 2 we show the MOND rotation curves of NGC 3198 when the galaxy is assumed to be at distances of $10 \mathrm{Mpc}$, 12.5 Mpc and 13.8 Mpc. Again, the MOND acceleration parameter is assumed to be the BBS value rescaled to the new distance scale, i.e., $0.9 \times 10^{-8} \mathrm{~cm} \mathrm{~s}^{-2}$.

The closest assumed distance, $10 \mathrm{Mpc}$, is roughly the Hubble law distance given the radial velocity of NGC 3198 with respect to the local group; it is also the least-square-fit distance if distance is left as a free parameter in the context of MOND. The distance of $13.8 \mathrm{Mpc}$ is the final Cepheid-based distance given by Freedman et al. (2001); and $12.5 \mathrm{Mpc}$ corresponds to the Cepheid distance less $10 \%$. The disc $M / L$ values in the $B$-band corresponding to the MOND fits at these various distances are also given in Fig. 2.

Here we see that the MOND rotation curve for a distance of $10 \mathrm{Mpc}$ is essentially a perfect fit to the observed curve. At the distance of $12.5 \mathrm{Mpc}$, the MOND curve is less than a perfect match, but, nowhere that the rotation curve is well measured, does the predicted rotation curve deviate by more than $5 \mathrm{~km} \mathrm{~s}^{-1}$ from the curve derived from the observed velocity field. This is typically within the difference in the rotation curves derived from the two sides of the galaxies considered separately-a sensible estimate of the uncertainties (the error bars are formal errors determined from the tilted ring fitting procedure).

At the Cepheid distance of $13.8 \mathrm{Mpc}$, the MOND rotation curve deviates in the same sense but by now up to $-10 \mathrm{~km} \mathrm{~s}^{-1}$ in the inner regions $\left(8-14 \mathrm{kpc}\right.$ ) and by $+10 \mathrm{~km} \mathrm{~s}^{-1}$ in the outer regions $(30-40 \mathrm{kpc})$. The reason for the deteriorating fit with increasing assumed distance is the relatively larger contribution of the gaseous component to the rotational velocity. The rotation curve of NGC 3198 in the outer regions $(r>20 \mathrm{kpc})$ is constant at about $150 \mathrm{~km} \mathrm{~s}^{-1}$. This would imply, in the context of MOND, that essentially the entire mass of the galaxy is 
Table 1. Galaxies with well-defined rotation curves and Cepheid-based distances.

\begin{tabular}{cccccccc}
\hline \hline Galaxy & $\begin{array}{c}D \\
(\mathrm{Mpc})\end{array}$ & $\begin{array}{c}L_{B} \\
\left(10^{10} L_{\odot}\right)\end{array}$ & $\begin{array}{c}M_{\text {gas }} \\
\left(10^{10} M_{\odot}\right)\end{array}$ & $\begin{array}{c}M_{\text {disc }} \\
\left(10^{10} M_{\odot}\right)\end{array}$ & $\begin{array}{c}M_{\text {disc }} / L_{B} \\
(1)\end{array}$ & $\begin{array}{c}M_{\text {bulge }} \\
\left(10^{10} M_{\odot}\right)\end{array}$ & $M_{\text {bulge }} / L_{B}$ \\
$(1)$ & $(2)$ & $(3)$ & $(4)$ & $(5)$ & $(6)$ & $(7)$ & $(8)$ \\
\hline NGC 2403 & $3.2 \pm 0.2$ & 0.82 & 0.4 & $1.34 \pm 0.03$ & 1.6 & & \\
NGC 2841 & $14.1 \pm 1.5$ & 4.60 & 2.7 & $29.70 \pm 4.3$ & 8.3 & 1.5 & 0.83 \\
NGC 3198 & $13.8 \pm 0.5$ & 2.44 & 1.6 & $2.63 \pm 0.1$ & 1.1 & & 1.8 \\
NGC 7331 & $14.7 \pm 0.6$ & 5.26 & 1.4 & $13.20 \pm 0.6$ & 2.0 & 5.7 & 1.8 \\
\hline
\end{tabular}

(2) The Cepheid-based distance from Freedman et al. (2001).

(3) The $B$-band luminosity (in $10^{10} L_{\odot}$ ) at the Cepheid distance.

(4) The total gas mass including primordial helium at the Cepheid distance.

(5) The total mass of the stellar disc from the MOND fit.

(6) The implied mass-to-light ratio of the stellar disc.

(7) The total mass of the stellar bulge in those two cases where a bulge is evident.

(8) The implied mass-to-light ratio of the stellar bulge.

enclosed within about $20 \mathrm{kpc}$, but this is obviously not the case given the significant surface density of neutral gas in the outer regions - contributing more than $50 \mathrm{~km} \mathrm{~s}^{-1}$ to the Newtonian rotation curve at the last measured point.

At a distance of $12.5 \mathrm{Mpc}$, the MOND rotation curve appears to be consistent with the observed curve (within the likely errors of the method for estimating rotation curves from $21 \mathrm{~cm}$ line data). Although this distance is formally $2 \sigma$ below the Cepheid-based distance, it is unclear if all systematic effects connected with this method are well-understood. It has been noted, for example, that for the galaxy NGC 4258 the kinematic water-maser-based distance is also about $10 \%$ less than the Cepheid-based distance (Maoz et al. 1999). The error budget of the Cepheid method is probably on the order of $10 \%$.

Sakai et al. (1999) have calibrated the T-F relation using 21 spiral galaxies with known Cepheid distances in five color bands: $B, V, R, I$, and $H$. If one places NGC 3198 on the mean $B$-band relation its distance should be $12.2 \mathrm{Mpc}$, while for the $I$-band this distance is $13.3 \mathrm{Mpc}$. Thus the Tully-Fisher distance is essentially consistent with the maximum MOND distance. Although the MOND rotation curve fit clearly prefers a somewhat smaller distance than the Cepheid-based distance, the idea is in no sense falsified by this well-determined rotation curve.

The I-band Tully-Fisher relation from Sakai et al. is shown in Fig. 3. The open points show the position of NGC 3198 when at a distance of $10.0 \mathrm{Mpc}, 12.5 \mathrm{Mpc}$, and $13.8 \mathrm{Mpc}$. It is evident that, given the scatter in the observed relation, it is impossible to distinguish between these possibilities although distances of 12.5 to $13.8 \mathrm{Mpc}$ are clearly preferred.

\section{2. $K^{\prime}$ band photometry}

One possible reason for the small deviation of the MOND curve from the observed curve at the Cepheid-based distance is that the $r$-band photometry is not a precise tracer of the stellar light distribution due to possible contamination by newly-formed stars and dust absorption. For this reason we have also considered recent near-infrared photometry of this galaxy.
An image of NGC 3198 in the $K^{\prime}$ band has been obtained by Rothberg et al. (2000) in order to calibrate the near infrared Tully-Fisher relation. The observations and initial stages of the data reduction, like sky-subtraction and flat-fielding are described in that paper. The detector was $1024 \times 1024$ square pixels of size $11^{\prime \prime} 68 \times 1$ 1.' 68 . Consequently the total image measures 28.7 arcmin along the sides and NGC 3198 which has a scale-length of approximately 1 arcmin fits completely within the image leaving ample margins of pure sky around the galaxy. Rothberg et al. (2000) derived a total brightness of $7.79 K^{\prime}$ magnitudes which translates to $3.4 \times 10^{10} L_{\odot}^{K^{\prime}}$ for a distance of $13.8 \mathrm{Mpc}$.

In Fig. 4 the image of the central regions of NGC 3198 is reproduced. Clearly discernible is a prominent bulge which is much less obvious in images at bluer wavelengths. As a consequence this central bulge region must be enshrouded in an appreciable amount of dust, which explains the reddening going inward. Surrounding the bulge appears to be a ring of spiral arm features with a light depression between the bulge and this ring.

To determine the radial luminosity profile, ellipses have been fitted to the image which provided the position and orientation of the galaxy (Fig. 5). As a next step the intensities have been averaged over elliptic annuli. In the inner regions the orientations of the annuli were equal to those determined by the ellipse fit, while for the intermediate and outer regions a constant position angle and ellipticity was adopted. The error of each radial intensity value was calculated by quadratically adding the error generated by sky-level variations and the noise appropriate for each annulus. The result is shown in Fig. 6. The radial profile in the $r$-band is also plotted in that figure, and one may notice that the photometry of the disc is of similar shape for the $r$ and $K^{\prime}$ bands.

It is without doubt that NGC 3198 has a bulge or central light concentration. A possible bulge/disc light decomposition is shown in Fig. 7. Here, it is assumed that the mass surface density is exactly proportional to the observed intensity level, and that the light and mass distribution are axisymmetric. For that case the bulge/disc decomposition illustrated in Fig. 7 is essentially a decomposition by eye. Here, it is further assumed that the stellar disc has a central hole with a radius 

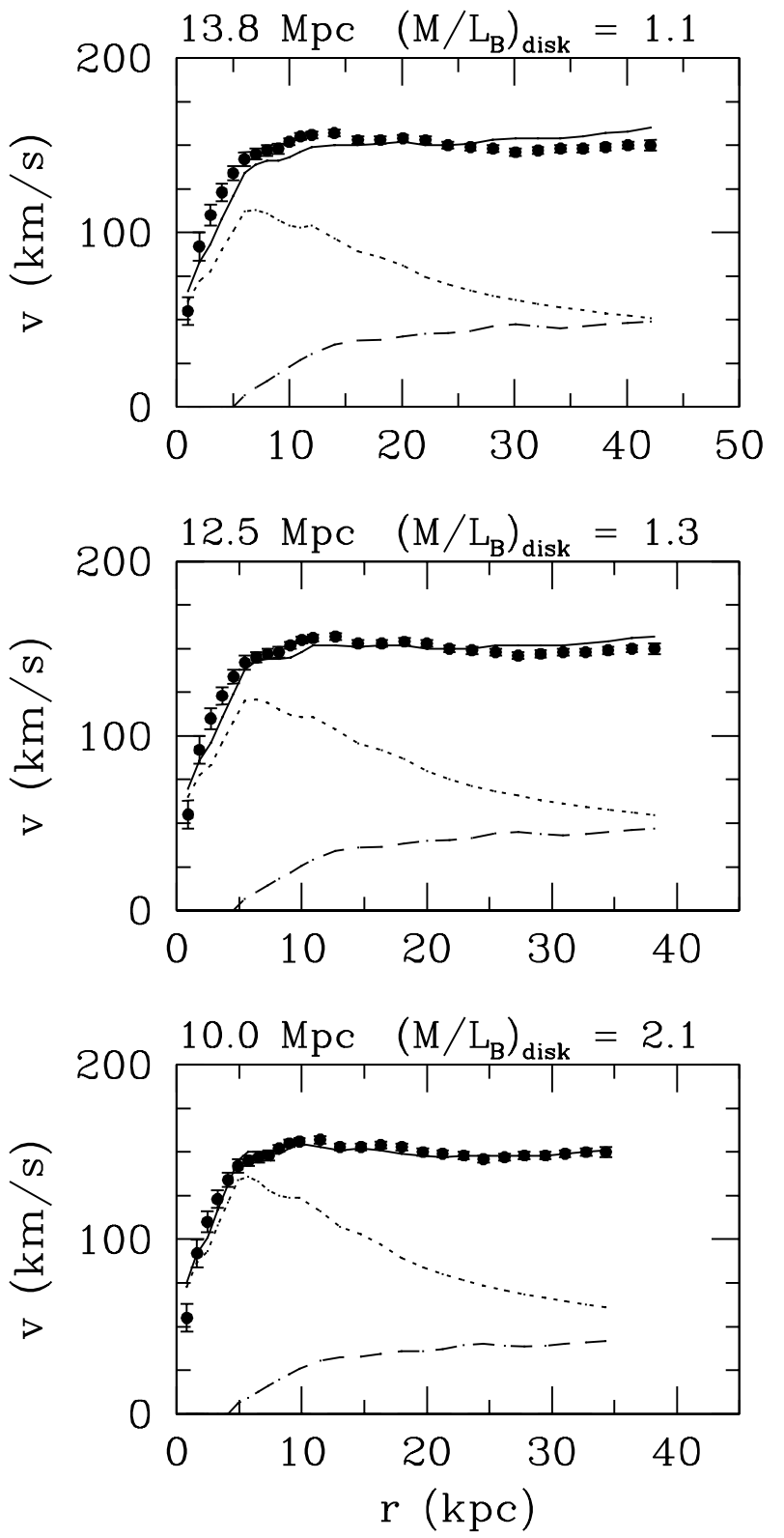

Fig. 2. MOND rotation curves for NGC 3198 assumed to be at various distances. The Cepheid-based distance is $13.8 \mathrm{Mpc}$. The dotted and dashed lines are the Newtonian rotation curves of the stellar and gas discs respectively.

corresponding to that of the light depression and that the bulge extends slightly beyond this radius.

The light depression might well be caused by the presence of a central bar. The influence of a bar on the radial velocity field of the gas is suggested in high-resolution $\mathrm{H} \alpha$ images of the galaxy, where characteristic distortions from circular motion are evident (Corradi et al. 1991). The light depression would then be due to a real deficiency of matter near the L4 and L5 Lagrangian points along the minor axis of the bar (Bosma 1978). In that case, the bar would be oriented nearly parallel to the line-of-sight and would not be photometrically conspicuous. Moreover, the bar would affect the derived rotation curve in the inner regions, or, at least, the interpretation of the rotation curve as a tracer of the radial force distribution. A bar aligned

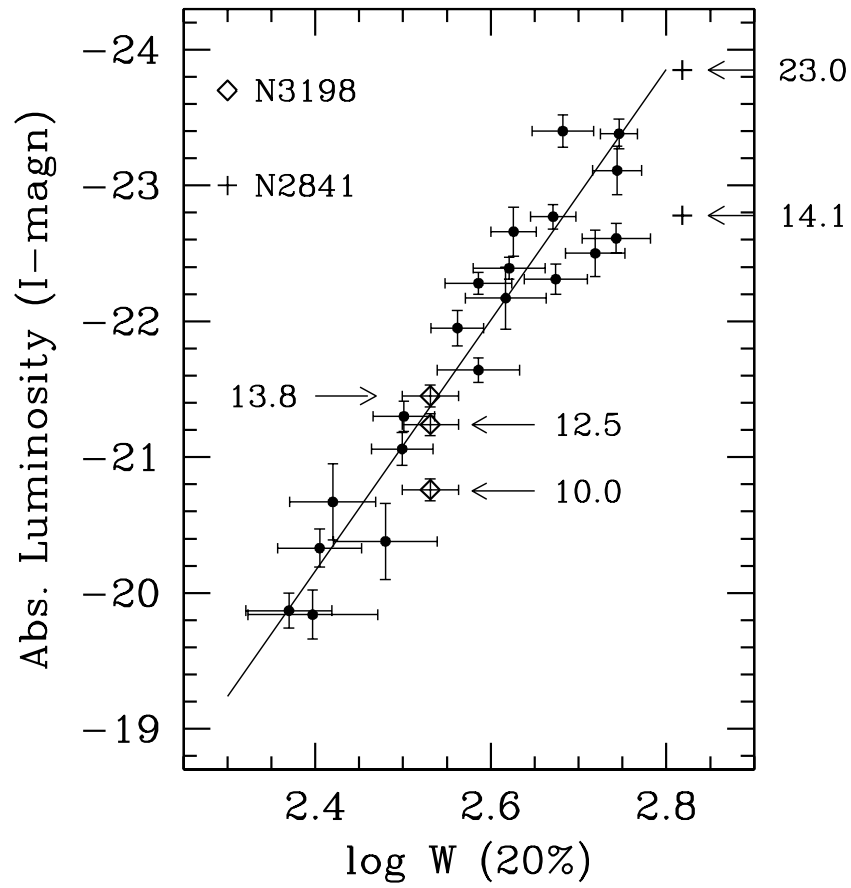

Fig. 3. The $I$-band Tully-Fisher relation for local calibrator galaxies with Cepheid-based distances. The positions of NGC 3198 (diamonds) and NGC 2841 (crosses), assumed to be at various distances, are indicated.

with the minor axis of the galaxy image would have the effect of increasing the apparent rotation velocities in the inner region (Teuben \& Sanders 1985); however, this would be significant only within the inner $30 \operatorname{arcsec}(\approx 2 \mathrm{kpc})$ and would have little influence upon the overall shape of the derived $21 \mathrm{~cm}$ line rotation curve.

Keeping this caveat in mind, we proceed using the decomposition depicted in Fig. 7: assuming a spherical bulge and disc with observed ellipticity, the total $K^{\prime}$ luminosity of $3.4 \times$ $10^{10} L_{\odot}^{K^{\prime}}$, is divided into $8.23 \times 10^{9}$ and $29.09 \times 10^{9} L_{\odot}^{K^{\prime}}$ for the bulge and disc respectively.

Because the scale-length of the disc in $K^{\prime}$ is nearly equal to the disc scale-length in the optical, it is not to be expected that the MOND fit will be much different from that for the $r$-band photometry. This is the case, as can be seen in Fig. 8 where the MOND rotation curve again has been determined at the Cepheid-based distance of $13.8 \mathrm{Mpc}$. Here, except for a spike in the central regions which is due to the bulge, the predicted rotation curve is essentially the same as derived from the $r$-band photometry; that is to say, the conclusions are unchanged by the near-infrared results.

\section{NGC 2841}

In Fig. 9 we show the MOND rotation curve for NGC 2841 compared to the observed curve at various assumed distances: 15.6 Mpc, which is the $1 \sigma$ upper limit on the Cepheid-based distance, $17 \mathrm{Mpc}$ which is $20 \%$ larger than the Cepheid-based distance, and $23 \mathrm{Mpc}$ which is the MOND-preferred distance. As in Fig. 2 the $M / L$ values for the disc and bulge are also given in the figure. 


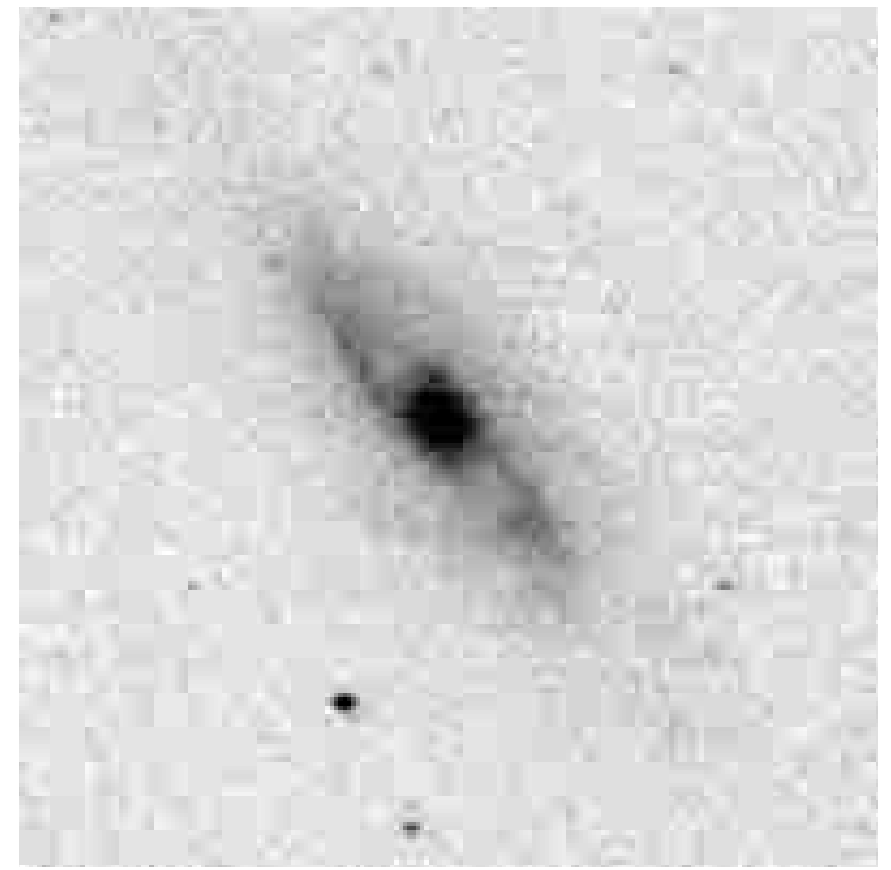

Fig. 4. The $K^{\prime}$ image of the central regions of NGC 3198. Brightness is represented by a linear gray-scale until $16.94 \mathrm{mag}$. $\operatorname{arcsec}^{-2}$ and black beyond this level. The size of the image is 5.6 by 5.6 arcmin. North is at the top, east to the left. NGC 3198 has a clear bulge in the nearinfrared surrounded by what seems to be a small light depression and then a ring of spiral arm features.

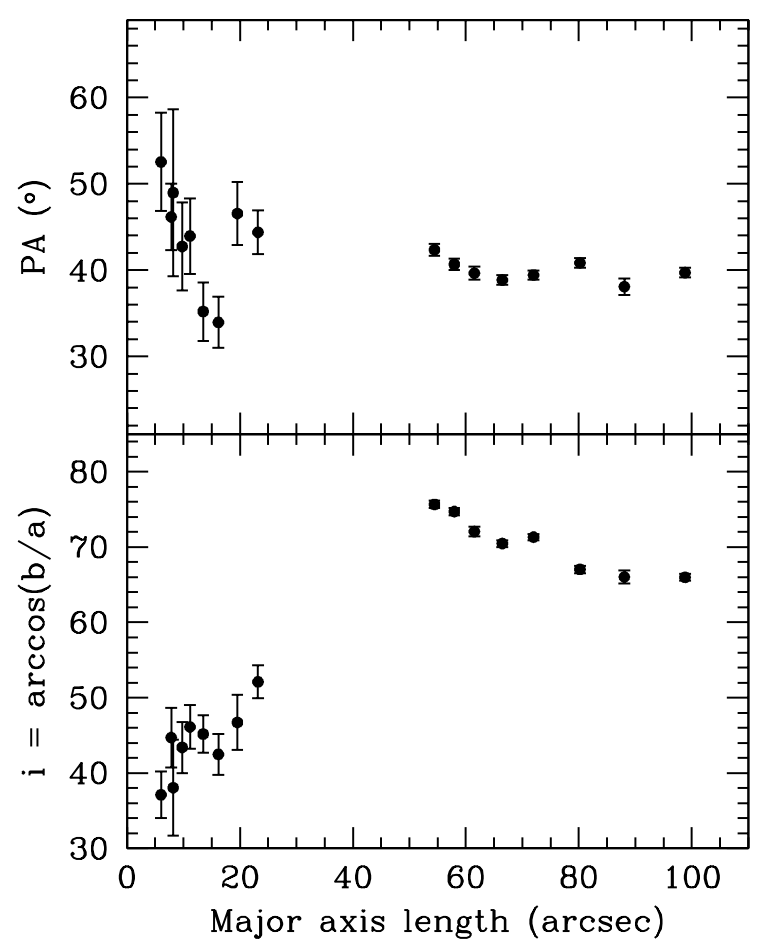

Fig. 5. The orientation and inclination of ellipses fitted to the $K^{\prime}$ band image of NGC 3198. The same fixed central positions have been adopted for all ellipses. The distinct signature of the central bulge (bar) is apparent.

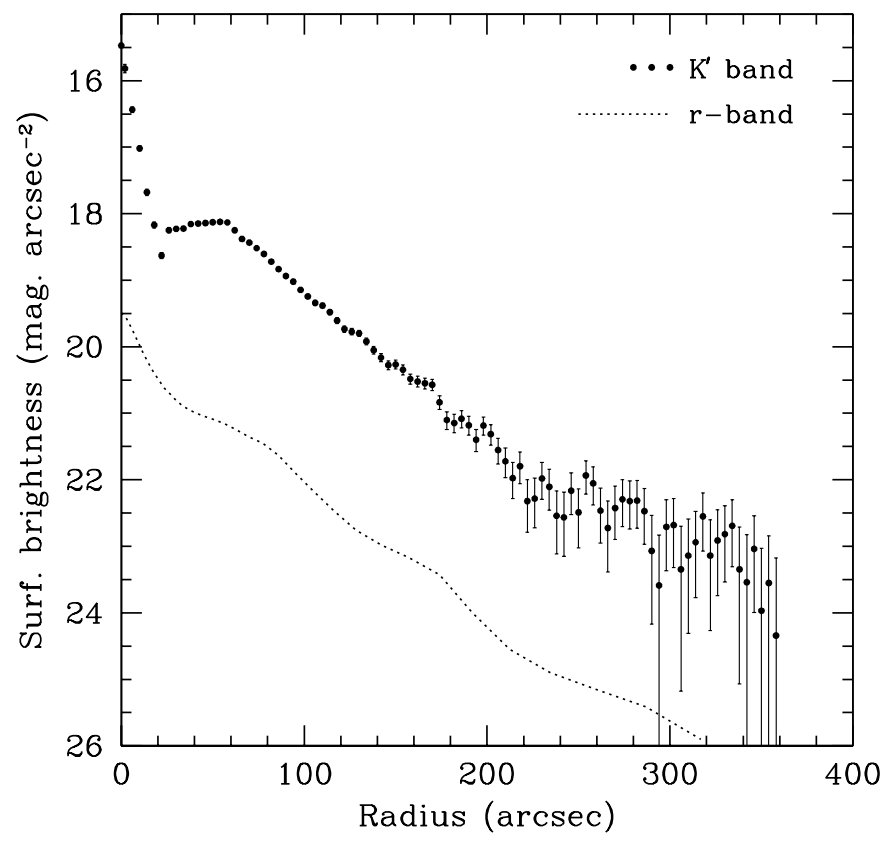

Fig. 6. Radial light profile in the $K^{\prime}$ band compared with that in the $r$-band (Kent 1987). The photometry of the disc is similar for both bands, however, in the near infrared the bulge is considerably brighter than in the optical.

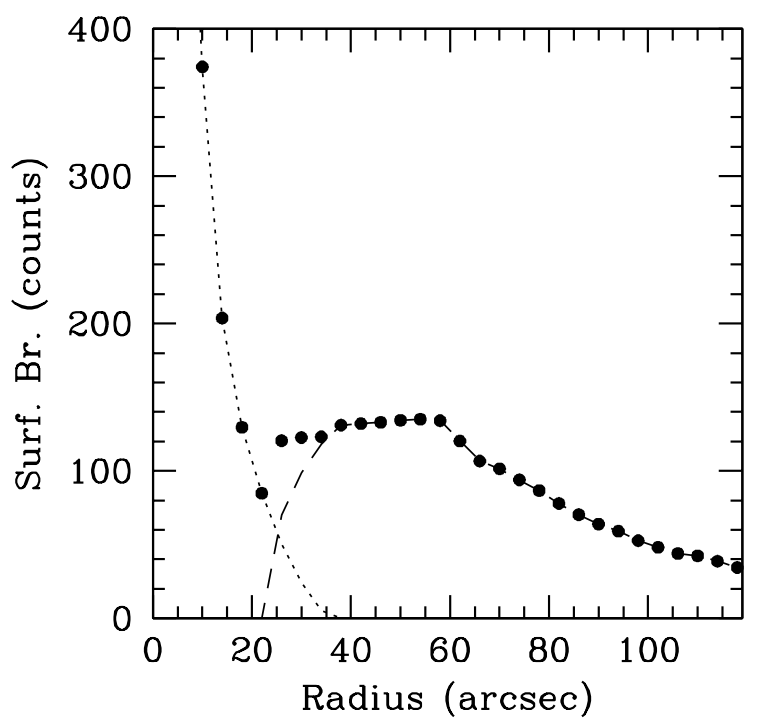

Fig. 7. Radial light profile in the central regions and a possible decomposition into a bulge (dotted line) and a disc (dashed line).

The rotation curve, as a tracer of the radial force distribution in this galaxy, is actually not as well-determined as that of NGC 3198. There is a significant warp in the outer regions which must be modelled by the tilted-ring technique, and this adds uncertainty to the derived rotation curve (see comments by Bosma 2002). None-the-less, it is clear that, while the Cepheid distance goes in the right direction (it is significantly larger than the Hubble based distance), it is not enough to bring the MOND-predicted rotation curve into agreement with the observed curve. Moreover, not only does the form of the predicted curve differ systematically from that observed, 


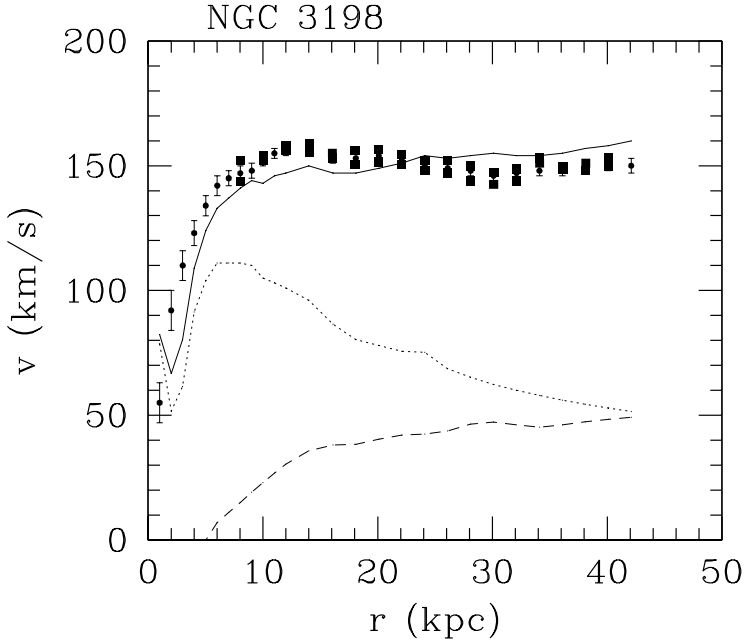

Fig. 8. The MOND rotation curve of NGC 3198 where the surface density distribution of the stellar component is taken to be traced by the $K^{\prime}$-band photometry using the decomposition shown in Fig. 7. The distance is Cepheid-based distance of $13.8 \mathrm{Mpc}$. The filled squares show the rotation curves derived for the two sides of the galaxy independently; this gives a better estimate of the errors.

but it is clear that the $M / L$ value for the disc is un-naturally large $\left(6.8 M_{\odot} / L_{\odot}\right)$ - larger than that required for the bulge (2.2).

Both of these problems are relieved somewhat if the distance is taken to be $20 \%$ larger than the Cepheid-based determination - at $17 \mathrm{Mpc}$. There are still systematic deviations in the form of the rotation curve, but these become large (in the outer regions), only where the gas layer of the galaxy is observed to be significantly warped. We may take this as a lower limit on the distance which would be compatible with MOND, although the disc $M / L$ does remain uncomfortably large $\left(5.9 M_{\odot} / L_{\odot}\right)$ and that of the bulge rather small (2.7).

Leaving distance as a free parameter in the fit yields a MOND-preferred distance of $23 \mathrm{Mpc}$, and here we see that the rotation curve fit is perfect with very reasonable implied $M / L$ values for the disc and bulge. This is entirely consistent with the distance implied by the Cepheid-calibrated TullyFisher relation as is also shown in Fig. 3. Taking the galaxy to be at the Cepheid distance of $14.1 \mathrm{Mpc}$, we see that the galaxy lies about one entire magnitude below the mean line of the TF relation. The distance implied by $I$-band TF relation is $24 \mathrm{Mpc}$.

There has been a recent supernova in NGC 2841 (SN 1999by), which is type Ia, i.e., the fundamental extragalactic "standard candle". However, if the galaxy is at the Cepheid distance of 14.1 Mpc, SN 1999by is one of the least luminous supernovae Ia ever observed, with a peak absolute magnitude of $M_{B}=-17.15 \pm 0.23$. Based upon an estimate of the decline-rate parameter $\left(\Delta m_{15} \approx 1.9\right)$ Garnivich et al. (2001) argue that this supernova is a peculiar low luminosity event, and they use this event and several others to recalibrate the Phillips relation (Phillips et al. 1999) between decline rate and peak luminosity. However, if we take the Phillips relation at face value then the peak luminosity of this object would be $M_{B}=-18.3$, which would imply that the distance to the galaxy would be $23.5 \mathrm{Mpc}$. It is interesting that an earlier SN event in
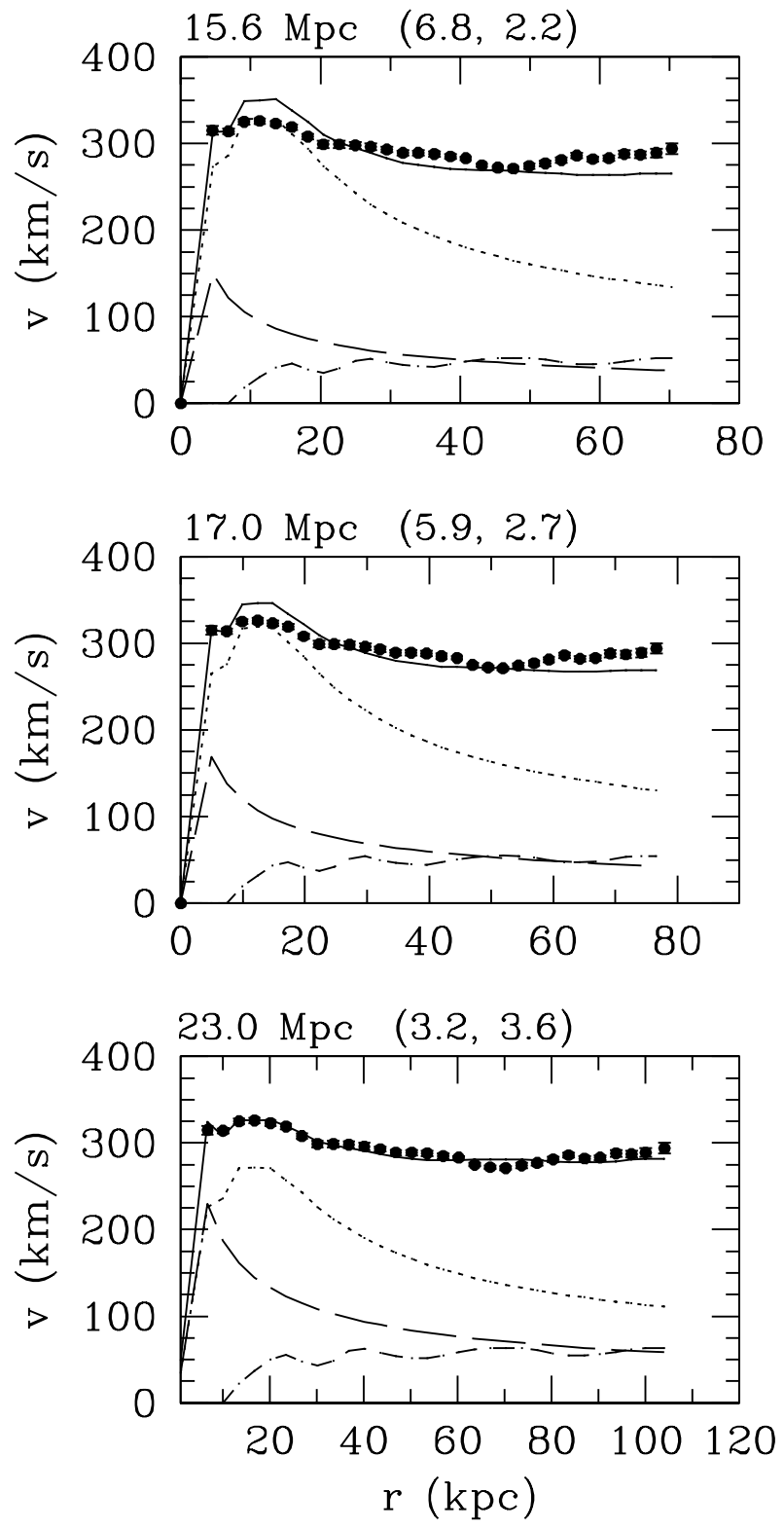

Fig. 9. MOND rotation curves for NGC 2841 at various distances ranging from the one-sigma upper limit on the Cepheid-based distance $(15.6 \mathrm{Mpc})$ to the Tully-Fisher and SNIa distance $(23 \mathrm{Mpc})$. The $M / L_{\mathrm{B}}$ values for the disc and bulge respectively are given in parenthesis.

NGC 2841, SN 1957A, would be, if the galaxy is at the Cepheid distance, the faintest supernova type Ia ever observed $\left(M_{B}=\right.$ $-16.4)$. It is curious that this galaxy only seems to provide subluminous supernovae.

The deviation of the galaxy from the TF relation and the abnormally low peak powers of supernovae, suggest that the Cepheid distance to this object may be substantially too low. It has been argued that the Cepheid method may be adversely affected by blending: the true apparent brightness of Cepheids is enhanced by blending with the light of nearby stars. This would lead to an underestimate of distances based upon the periodluminosity relation, and would affect, in particular, the more distant objects (see Paczyński \& Pindor 2001 for a discussion 
of these points). All we can conclude, at the moment, is that the MOND-preferred distance to NGC 2841 remains significantly larger than the present Cepheid-based distance.

\section{Conclusions}

The main conclusions of this paper can be summarized as follows:

1. For the galaxies NGC 2403 and NGC 7331, MOND rotation curves agree acceptably with the observed curves when these galaxies are taken at the Cepheid-based distance.

2. For NGC 3198 at the Cepheid-based distance of $13.8 \mathrm{Mpc}$, the MOND curve shows small $\left(<10 \mathrm{~km} \mathrm{~s}^{-1}\right)$ but significant systematic deviations from the observed curve.

3. If the distance to NGC 3198 is taken to be $12.5 \mathrm{Mpc}$, or $10 \%$ less than the Cepheid-based distance, the MOND curve is an acceptable representation of the observed curve. This lower distance is probably within the uncertainties of the Cepheid method.

4. These conclusions are unaltered by utilizing recent nearinfrared photometry of NGC 3198 which does show evidence for a small central bulge and bar component.

5. For NGC 2841, the rotation curve predicted by MOND when the galaxy is taken to be at the upper limit on the Cepheid-based distance $(15.6 \mathrm{Mpc})$ remains inconsistent with the observed curve, with systematic deviations of more than $30 \mathrm{~km} \mathrm{~s}^{-1}$.

6. The smallest distance for which the MOND curve is compatible with the observed curve (given the uncertainties involved in the tilted ring technique for modelling warps), is $17 \mathrm{Mpc}$ or $20 \%$ larger than the Cepheid-based distance. The preferred MOND distance is $23 \mathrm{Mpc}$.

7. The TF distance to NGC 2841, based upon the Cepheidre-calibrated TF relation is $24 \mathrm{Mpc}$. If the distance to this galaxy is really $14.1 \mathrm{Mpc}$, then it would deviate from the mean $I$-band TF relation by more than 1 mag.

8. NGC 2841 has been the host of a type Ia supernova, 1999by. If this galaxy is at the Cepheid-based distance, this would be one of the least intrinsically luminous supernovae ever observed. Calibrating the peak luminosity by the Phillips relation, the $\mathrm{SN}$-based distance is $23.5 \mathrm{Mpc}$.

It is clear that NGC 2841 remains a critical case for MOND. The discrepancy between Cepheid-based distance and both the TF and SNIa based distances to NGC 2841 suggests that there may be a problem with the derived Cepheid-based distance.

In general, it is evident that accurate distance determinations to nearby galaxies are extremely relevant to the question of the viability of MOND. MOND, as a modification of Newtonian dynamics attached to an acceleration scale, is far more fragile than the dark matter hypothesis in this regard. It would be useful to obtain more Cepheid-based distances to the sample of galaxies with well-observed rotation curves. Particularly useful would be a Cepheid distance estimate to the Ursa Major cluster as many of these galaxies have wellmeasured rotation curves and near-infrared photometry.

Acknowledgements. We are grateful to M. Milgrom for a critical reading of the manuscript and we thank the referee A. Bosma, for giving useful comments which have improved the paper.

\section{References}

Begeman, K. G. 1987, Ph.D. Dissertation, Univ. of Groningen

Begeman, K. G., Broeils, A. H., \& Sanders, R. H. 1991, MNRAS, 249, 523 (BBS)

Bosma, A. 1978, Ph.D. Dissertation, Univ. of Groningen

Bosma, A. 2002, in The Dynamics, Structure and History of Galaxies, ed. G. da Costa, \& H. Jerjen, ASP Conf. Ser., 273, 223

Corradi, R. L. M., Boulesteix, J., Bosma, A., et al. 1991, A\&A, 244, 27

Freedman, W. L., \& Madore, B. F. 1988, ApJ, 326, 691

Freedman, W. L., Madore, B. F., Gibson, B. K., et al. 2001, ApJ, 553, 47

Garnavich, P. M., Bonanos, A. Z., Jha, S., et al. 2001 [astro-ph/0105490]

Hughes, S. M. G., Han, M., Hoessel, J., et al. 1998, ApJ, 501, 32

Hunter, D. A., Rubin, V. C., \& Gallagher, J. S. 1986, AJ, 91, 1086

Kelson, D. D., Illingworth, G. D., Saha, A., et al. 1999, ApJ, 514, 614

Kent, S. M. 1987, AJ, 93, 106

McGaugh, S. S., \& de Blok, W. J. G. 1998, ApJ, 499, 66

Macri, L. M., Calzetti, D., Freedman, W. L., et al. 2001, ApJ, 549, 721

Maoz, E., Newman, J. A., Ferrarese, L., et al. 1999, Nature, 401, 351

Milgrom, M. 1983, ApJ, 270, 365

Paczynski, B., \& Pindor, B. 2000, ApJ, 533, L103

Phillips, M. M., Lira, P., Suntzeff, N. B., et al. 1999, ApJ, 118, 1766

Rothberg, B., Saunders, W., Tully, R. B., \& Witchalls, P. L. 2000, ApJ, 533, 781

Sakai, S., Mould, J. R., Hughes, S. M. G., et al. 2000, ApJ, 529, 698

Sanders, R. H. 1996, ApJ, 473, 117

Sanders, R. H., \& Verheijen, M. A. W. 1998, ApJ, 503, 97

Teuben, P. J., \& Sanders, R. H. 1985, MNRAS, 212, 257

Trimble, V. 1987, ARA\&A, 25, 425

Tully, R. B., \& Fisher, J. R. 1977, A\&A, 54, 661

Tully, R. B., \& Pierce, M. J. 2000, ApJ, 533, 744

van Albada, T. S., Bahcall, J. N., Begeman, K., \& Sancisi, R. 1985, ApJ, 295, 305

van der Kruit, P. C., \& Searle, L. 1981, A\&A, 95, 105 